(C) 2005 IEEE. Personal use of this material is permitted. Permission from IEEE must be obtained for all other uses, in any current or future media, including reprinting/republishing this material for advertising or promotional purposes, creating new collective works, for resale or redistribution to servers or lists, or reuse of any copyrighted component of this work in other works. 


\title{
Improved Measurement with 2D Rotating Fluxes Considering the Effect of Internal Field
}

\author{
Jin Jiang ZHONG, Jian Guo ZHU, Senior Member, IEEE, YouGuang GUO, Member, IEEE, \\ and Zhi Wei LIN
}

\begin{abstract}
-this paper analyzes the effect of internal field on the measurement under rotating fluxes. Due to the effect, the angular error cannot be corrected completely, causing the asymmetry of $H$ loci and the discrepancy of rotation core loss between two opposite rotating directions. A numerical averaging method is employed to eliminate the effect of internal field. Finally, a type of SMC material is systematically measured and corrected.
\end{abstract}

Index Terms -internal field, misalignment angle, field-metric method, magnetization, SST tester.

\section{INTRODUCTION}

$\mathrm{S}$ INCE the rotational power losses in electrical sheet steels were quantitatively measured for the first time a great number of measuring techniques and testing systems have been developed for rotational B-H relationship and core loss measurement. Among these testers, the square single sheet tester (SST) initiated by Brix and Hempel [1] appears to be the most favorable, because it is more flexible to control the rotating magnetic flux pattern and the magnetic field in the sample is more uniform and hence higher accuracy of measurement.

Some phenomena were reported previously by using the field metric method in SST [2-9], that there were significant asymmetry of $\mathbf{H}$ loci, and the big discrepancy of rotational core loss in the clockwise and the anti-clockwise directions. In additional, T. Sasaki et al [3] observed that when the flux density is above a critical value, one loss component increases rapidly and the other decreases more and more and at last shows a negative loss. Salz and Hempel [5] found that both the alternating and rotational components of the core loss under an elliptical rotating field had negative values.

These phenomena have not so far been fully understood yet. One of the explanations in the previous literature was attributed to the misalignment of the $\mathbf{H}$ sensors and the asymmetry in the devices [2,6,7,9]. However, the calculated correction for the angular error of $\mathbf{H}$ sensors could not completely eliminate the asymmetry and discrepancy in spite of various attempts [4].

Another explanation was that because the silicon iron alloy has very large crystal anisotropy energy, the rotation of

Manuscript received February 7, 2005.

The authors are with the Center for Electrical Machine and Power Electronics, Faculty of Engineering, University of Technology, Sydney, NSW 2007 Australia (telephone: +61-2-95141268, e-mail: zhong@eng.uts.edu.au, joe@eng.uts.edu.au, youguang@eng.uts.edu.au, jacklin@eng.uts.edu.au). magnetization is difficult to take place [3]. On the contrary, the magnetization lying in an unfavorable direction requires the minus energy that was a generation of energy, in order to return to the easy direction. Alinejad-Beromi et al [8] also suggested that the losses depend on the direction of rotation. They argued that in their experiment, the effect of slight misalignment of the axes of the $\mathbf{H}$ coils was examined under clockwise and anti-clockwise rotations of the magnetic field without sample presented. The measured field components agreed well, within $\pm 0.5 \%$ on the coil axes and with a slightly greater difference of $\pm 1.35 \%$ midway between the axes, while the difference between the rotational losses in two rotating directions was very large. It is from $12 \%$ to $30 \%$ for different materials. They ascribed the difference in loss to a genuine difference in the magnetization process occurring in each case due to the structure of the material. However, so far these extrapolations lack theoretical support.

In this paper, this phenomenon is to be explained based on an analysis of the effects of internal field on the measurement under rotating fluxes. The authors believe that the real misalignment angles of $\mathbf{H}$ sensing coils cannot exactly be evaluated due to the effect of internal field, and therefore the angular error cannot be corrected completely.

\section{MEASUREMENT InSTRUMENT}

The square sample single sheet tester used for the measurement of the magnetic properties of soft magnetic composite materials under 2D magnetic excitations was developed and modified at University of Technology, Sydney. Fig.1 illustrates schematically the square sample single sheet tester and the whole testing system.

To determine the total core loss and B-H relationship in two dimensions in a sample, the flux density inside the sample and the surface field strength should be measured accurately. In the testing presented in this paper, 2D Rogowski-Chattock $\mathbf{H}$ coils are employed measuring for the surface field strength. Since the coils can be installed right and close on the sample surface, the correct value of the surface field strength can be picked up. B-coils are used for measuring the flux density because of its high accuracy. For measurement of rotational core loss, the field-metric method is employed as the field-metric method features high accuracy and great versatility. Moreover, the measured instantaneous $\mathbf{H}$ and $\mathbf{B}$ values can yield additional information, such as various loss contributions, the loci of $\mathbf{H}$ and $\mathbf{B}$ vectors, and harmonics, etc. 


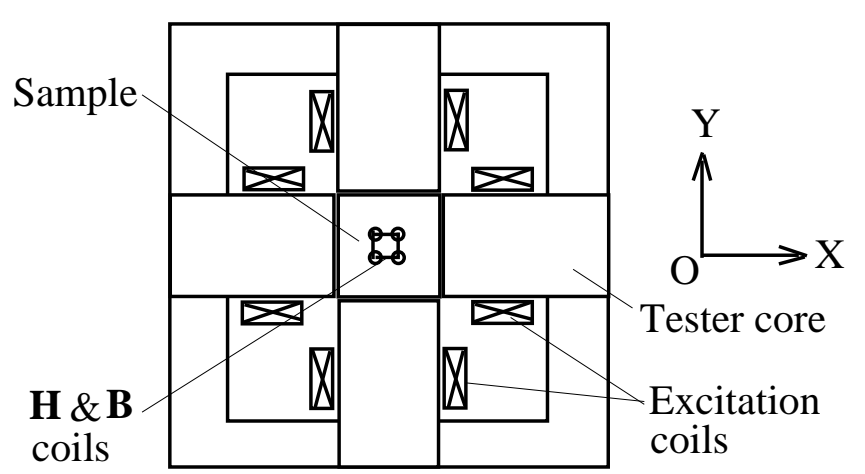

(a)

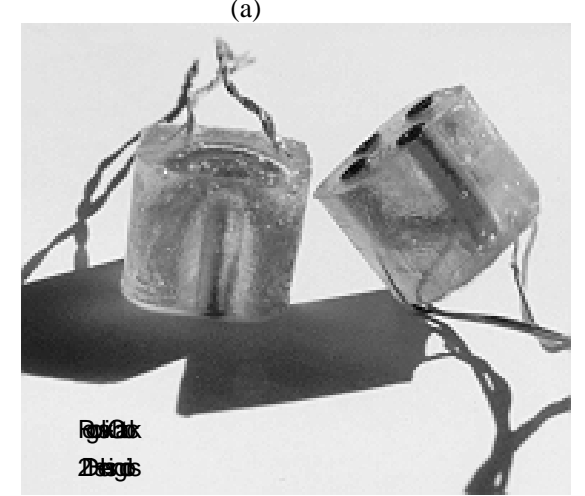

(b)

Fig. 1. Schematic illustrations of SST tester, (a) the square specimen single sheet tester, and (b) Two dimensional Rogowski-Chattock $\mathbf{H}$ coils

\section{EFFECT OF INTERNAL FIELD ON MEASUREMENT}

If the axes of the $\mathbf{H}$ sensing coils are misaligned with the axes of the excitation coils of the tester, or not perpendicular to each other, the measured values will not be the true components on the $\mathrm{X}$ and $\mathrm{Y}$ axes of the magnetic field. Theoretically, the error caused by this misalignment can be eliminated by using the coordinate transformation. However, it is not easy do to so in practice.

In our experiment for measuring the soft composite magnetic (SMC) material, the misalignment of the $\mathbf{H}$ coils was initially evaluated and corrected at low flux densities, e.g. $B=0.13 T$. However, the correction of the misalignment of the $\mathbf{H}$ coils became "invalid" at high flux densities, and the misalignment angles varied with the magnetization $\mathbf{M}$, as shown in Fig. 2. For a clear view of the discrepancy, different scales are used for the $\mathrm{X}$ and $\mathrm{Y}$ axes.

To study these phenomena, a lot of experiments have been carried out at different saturation levels, and the results were identical under the same condition. Since the $\mathbf{H}$ sensing coils are physically fixed during the test and the effect of magnetostriction of this kind of materials is negligible, it can be extrapolated that the variation of the misalignment angles of the $\mathbf{H}$ coils with the saturation level must be related to certain intrinsic mechanism of magnetization in the magnetic material under test. One possible reason is the effect of the internal field on the evaluation and the correction of misalignment angles of $\mathbf{H}$ sensing coils. Internal field that relates to the interaction and demagnetization fields inside the specimen, is a function of magnetization, denoted as $\mathbf{H}_{\mathbf{i}}=f(\mathbf{M})$, thus the above mentioned phenomena are also directly attributed to the effect of magnetization.

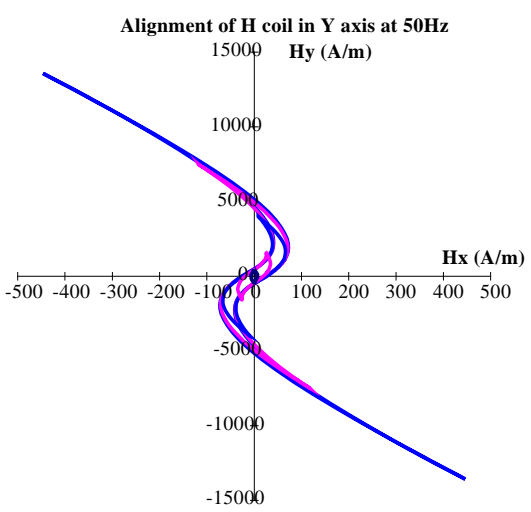

Fig. 2. Misalignment still exists after the correction for misalignment $\mathrm{H}$ sensing coils have been conducted by the coordinate rotation transformation method

In order to identify and correct the misalignment angles of the $\mathbf{H}$ sensors in an SST, alternating fields are applied to the $\mathrm{X}$ and $\mathrm{Y}$ axes respectively. In the ideal case of an isotropic material, subject to an alternating field $\mathbf{H}, \mathbf{M}$ is aligned with H. Consequently an actual misalignment angle of the sensing coils can be easily evaluated. In practice, due to the nonuniform distribution of magnetic particles and the asymmetric shape of the measured specimen, $\mathbf{M}$ always either leads or lags $\mathbf{H}$, and therefore, the internal field $\mathbf{H}_{\mathbf{i}}$ is not aligned with $\mathbf{H}$ as shown in Fig.3.

Fig.3 illustrates that an alternating field $\mathbf{H}$ applied in the $\mathrm{X}$ axis. Due to the effect of magnetization or internal field, the $\mathbf{H}$ vector is dragged away from the $\mathrm{X}$ axis. This leads to an extra or additional misalignment angle. The additional misalignment angle created by $f(\mathbf{M})$ depends on both the magnitude of $\mathbf{M}$ and its equilibrium direction.

The measured misalignment angles of $\mathbf{H}$ sensing coils can be simply expressed as

$\alpha_{i}(\mathbf{M})=\alpha_{i}+\alpha_{i}(f(\mathbf{M}))(\mathrm{i}=\mathrm{x}, \mathrm{y})$

where $\alpha_{i}$ is the actual misalignment angle, and $\alpha_{i}(f(\mathbf{M}))$ the additional misalignment angle, which depends on the variation of magnetization of the material under test.

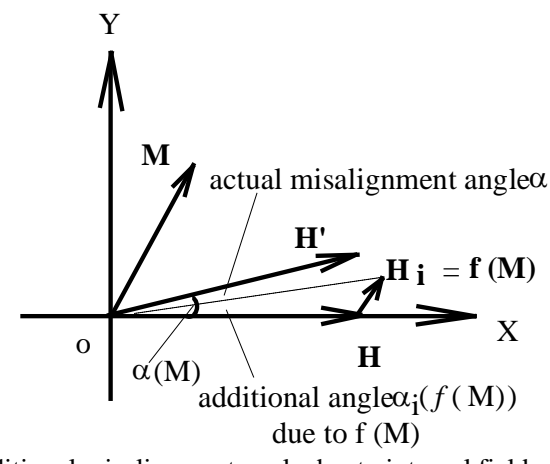

Fig. 3. Additional misalignment angle due to internal field. 
As mentioned above, due to the effect of the internal field $\mathbf{H}_{\mathbf{i}}$, it is quite difficult to obtain the correct (actual) misalignment angle, and thus the angular error of $\mathbf{H}$ sensing coils cannot be properly corrected. This will lead to an asymmetry in the measured $\mathbf{H}$ loci and a discrepancy in the rotational core losses calculated from the $\mathbf{H}$ loci of the two opposite rotating directions.

\section{Measuring Results Using an Averaging Method}

To eliminate the error caused by the additional misalignment angle, it is essential to evaluate $\mathbf{H}_{\mathbf{i}}$. In practice, it is not easy to get the functional relationship between $\mathbf{H}_{\mathbf{i}}$ and $\mathbf{M}$ for a specific magnetic material specimen. Alternatively, for the small misalignment angles, a convenient numerical method to correct the $\mathbf{H}$ locus for a given $\mathbf{B}$ locus was derived based on coordinate transformation, by averaging point by point of the $\mathbf{H}$ loci in two opposite rotating directions [11]. Sequentially the averaging method was employed to calculate the corresponding rotational core loss. Although a similar averaging method was proposed elsewhere [10], it should be indicated that the internal field and its effect on measurement were not considered and discussed in the previous literature.

Three square samples of SOMALOY ${ }^{\mathrm{TM}} 500$ soft magnetic composite material were tested. Figs.4(a) and (b) depict the $\mathbf{B}$ and $\mathbf{H}$ loci of a $50 \times 50 \times 1.25 \mathrm{~mm}$ sample corresponding to various circular rotating flux density vectors in clockwise and anti-clockwise directions. The broken lines in (b) show the $\mathbf{H}$ loci in anti-clockwise direction, and the solid lines are the averaged results.

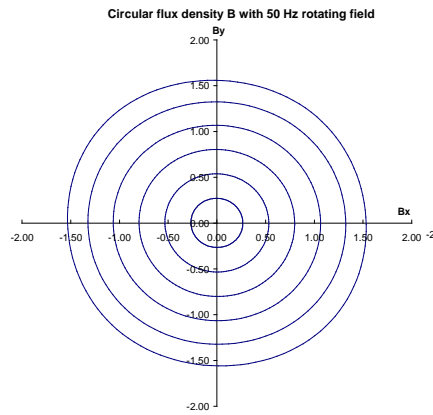

(a)

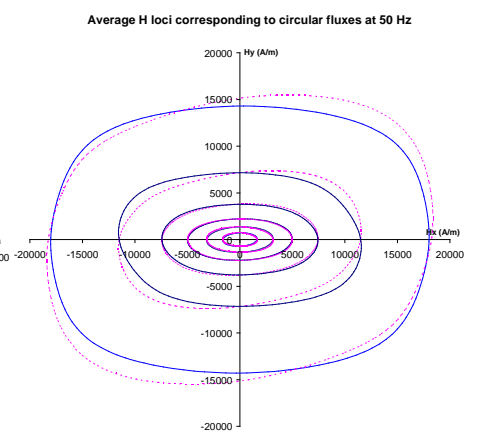

(b)
Fig. 4. Loci of (a) $\mathbf{B}$ and (b) $\mathbf{H}$ vectors in sample $\mathrm{d}$ with circular rotating flux density vector at $50 \mathrm{~Hz}$.

Fig. 5 illustrates the rotational core losses at the different saturation levels of flux density. The solid line represents the averaged core loss which looks reasonable, and the broken lines show the rotational core losses in the clockwise and anticlockwise directions respectively.

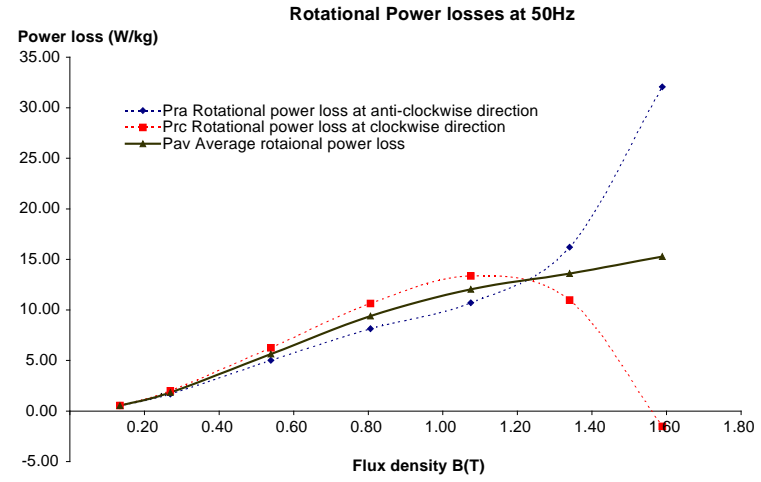

Fig.5 Rotational core losses at the different saturation levels of flux density.

\section{CONCLUSION}

The asymmetry in the $\mathbf{H}$ loci and discrepancy in the rotational core losses for the rotating flux density vectors in the two opposite directions can be explained by the effect of internal field on the evaluation and correction of misalignment of $\mathbf{H}$ sensing coils. The proposed averaging numerical method can provide more sensible experimental results.

\section{REFERENCES}

[1] W. Brix, K.A. Hempel, and W. Schroeder, "Method for the measurement of rotational power loss and related properties in electrical steel sheets,” IEEE Trans. Magn. Vol. 8, Nov. 1982, pp. 1469-1471.

[2] W. Brix, K.A. Hempel, and F.J. Schulte, "Improved method for the investigation of the rotational magnetization process in electrical steel sheets,” IEEE Trans. Magn. Vol. 20, Sept. 1984, pp. 1708-1710.

[3] T. Sasaki, M. Imamura, S. Takada, and Y. Suzuki, "Measurement of rotational power losses in silicon-iron sheets using wattmeter method," IEEE Trans. Magn. Vol. 21, Sept. 1985, pp. 1918-1920.

[4] J. Sievert, J. Xu, L. Rahf, M. Enokizono, and H. Ahlers, "Studies on the rotational power loss measurement problem,” Anales de Fisica Serie B, Vol. 86, 1990, pp. 35-37.

[5] W. Salz and K.A. Hempel, "Anisotropy of grain-oriented steel sheet under various elliptical field conditions,” J. Appl. Phys. Vol. 70, Nov. 1991, pp. 6268-6270.

[6] J. Sievert, "Studies on the measurement of two dimensional magnetic phenomena in electrical sheet steel at PTB," First Int. Workshop on Magnetic Properties of Electrical Sheet Steel under Two-Dimensional Excitation, Proceedings of the 93 PTB-Seminar, PhysikalischTechnische Bundesanstalt (PTB), Braunschweig, Germany), Sept., 1991, pp. 102-116.

[7] W. Salz and K.A. Hempel, "Which field sensors are suitable for a rotating flux apparatus," First Int. Workshop on Magnetic Properties of Electrical Sheet Steel under Two-Dimensional Excitation, Proceedings of the 93 PTB-Seminar, Physikalische Bundesanstalt (PTB), Braunschig, Germany, 1991, pp. 117-126.

[8] Y. Alinejad-Beromi, A.J. Moses, and T. Meydan, "New aspects of rotational field and flux measurement in electrical steel," Journal of Magnetism and Magnetic Materials, Vol. 112, 1992, pp. 135-138.

[9] J. Sievert, "On measuring the magnetic properties of electrical sheet steel under rotational magnetization," Journal of Magnetism and Magnetic Materials, Vol. 112, 1992, pp. 50-57.

[10] J.J. Zhong, "Measurement and modelling of magnetic properties of materials with rotating fluxes," PhD thesis, Uni. of Tech., Sydney, Dec. 2002. 